Research Article

\title{
Composition Semigroups on Weighted Bergman Spaces Induced by Doubling Weights
}

\author{
Fanglei Wu \\ Department of Mathematics, Shantou University, Shantou, Guangdong 515063, China \\ Correspondence should be addressed to Fanglei Wu; fangleiwu1992@gmail.com
}

Received 31 December 2020; Accepted 20 January 2021; Published 4 February 2021

Academic Editor: Dimitri Mugnai

Copyright (C) 2021 Fanglei Wu. This is an open access article distributed under the Creative Commons Attribution License, which permits unrestricted use, distribution, and reproduction in any medium, provided the original work is properly cited.

We prove that composition semigroups are strongly continuous on weighted Bergman spaces with doubling weights. Point spectra and compact resolvent operators of infinitesimal generators of composition semigroups are characterized.

\section{Introduction}

Let $\mathscr{H}(\mathbb{D})$ denote the space of analytic functions in the unit disc $\mathbb{D}=\{z \in \mathbb{C}:|z|<1\}$. For a nonnegative function $\omega \in L^{1}([0,1))$, the extension to $\mathbb{D}$, defined by $\omega(z)=\omega(|z|)$ for all $z \in \mathbb{D}$, is called a radial weight. For $0<p<\infty$ and a radial weight $\omega$, the weighted Bergman space $A_{\omega}^{p}$ consists of $f \in \mathscr{H}(\mathbb{D})$ such that

$$
\|f\|_{A_{\omega}^{p}}^{p}=\int_{\mathbb{D}}|f(z)|^{p} \omega(z) d A(z)<\infty,
$$

where $d A(z)=d x d y / \pi$ is the normalized Lebesgue area measure on $\mathbb{D}$. As usual, $A_{\alpha}^{p}$ stands for the classical weighted Bergman space induced by the standard radial weight $\omega(z)=\left(1-|z|^{2}\right)^{\alpha}$, where $-1<\alpha<\infty$.

For a radial weight $\omega$, write $\widehat{\omega}(z)=\int_{|z|}^{1} \omega(s) \mathrm{d} s$ for all $z \in \mathbb{D}$. In this paper, we always assume $\widehat{\omega}(z)>0$, for otherwise $A_{\omega}^{p}=\mathscr{H}(\mathbb{D})$ for each $0<p<\infty$. A weight $\omega$ belongs to the class $\widehat{\mathscr{D}}$ if there exists a constant $C=C(\omega) \geq 1$ such that $\widehat{\omega}(r) \leq C \widehat{\omega}(1+r / 2)$ for all $0 \leq r<1$. For more knowledge about those Bergman spaces, see [1-4] and the reference therein.

A family $\left(\varphi_{t}\right)_{t \geq 0}$ of analytic self-maps of the unit disk $\mathbb{D}$ in the complex plane $\mathbb{C}$ is said to be a semigroup if the following conditions hold:

(i) $\varphi_{0}$ is the identity map of $\mathbb{D}$

(i) $\varphi_{t}{ }^{\circ} \varphi_{\mathrm{s}}=\varphi_{\mathrm{t}+s}$, for $t, s \geq 0$ (ii) For each $z \in \mathbb{D}, \varphi_{t}(z) \longrightarrow z$, as $t \longrightarrow 0^{+}$

A semigroup $\left(\varphi_{t}\right)_{t \geq 0}$ is said to be trivial if each $\varphi_{t}$ is the identity of $\mathbb{D}$. The infinitesimal generator of $\left(\varphi_{t}\right)_{t \geq 0}$ is defined as the function

$$
G(z)=\lim _{t \mapsto 0^{+}} \frac{\varphi_{t}(z)-z}{t}, \quad z \in \mathbb{D} .
$$

For any nontrivial semigroup $\left(\varphi_{t}\right)_{t>0}$, there exist a point $b \in \overline{\mathbb{D}}$ and an analytic function $P: \mathbb{D} \mapsto \mathbb{C}$ with $\operatorname{Re} P \geq 0$ such that

$$
G(z)=(\bar{b} z-1)(z-b) P(z)
$$

Refer [5] for the details. Representation (3) is unique, and the point $b$ is said to be the Denjoy-Wolff point of $\left(\varphi_{t}\right)_{t \geq 0}$.

Notice that each semigroup $\left(\varphi_{t}\right)_{t \geq 0}$ gives rise to a semigroup $\left(C_{t}\right)_{t \geq 0}$ consisting of composition operators on $H(\mathbb{D})$, the set of analytic functions on $\mathbb{D}$, where

$$
C_{t}(f):=C_{\varphi_{t}} f=f^{\circ} \varphi_{t}, \quad f \in H(\mathbb{D}) .
$$

Given a semigroup $\left(\varphi_{t}\right)_{t \geq 0}$ and a Banach space $X$ of analytic functions on $\mathbb{D}$, we say that $\left(\varphi_{t}\right)_{t \geq 0}$ generates a strongly continuous composition operator on $X$ if $C_{t}$ is bounded on $X$ and

$$
\lim _{t \rightarrow 0^{+}}\left\|C_{t} x-x\right\|_{X}=0 \quad \text { for all } x \in X \text {. }
$$


$\left(C_{t}\right)_{t \geq 0}$ is called uniformly continuous on $X$ if $\lim _{t \rightarrow 0^{+}}\left\|C_{t}-I\right\|=0$, where $I$ is the identity map on $X$. The infinitesimal generator of a strongly continuous semigroup $\left(C_{t}\right)_{t \geq 0}$ on a Banach space $X$ is the operator

$$
\Gamma x:=\lim _{t \longrightarrow 0^{+}} \frac{C_{t}(x)-x}{t},
$$

which is densely defined for every $x$ in the domain

$$
D(\Gamma):=\left\{x \in X: \lim _{t \rightarrow 0^{+}} \frac{C_{t}(x)-x}{t} \text { exists }\right\} .
$$

Refer [6] for more information about operator semigroup.

In 1978, Berkson and Porta [5] initially studied the strong continuity of semigroups of composition operators acting on the classical Hardy space $H^{P}(\mathbb{D})$. They proved that for $1 \leq p<\infty,\left(C_{t}\right)_{t \geq 0}$ is strongly continuous on $H^{p}$. Later, Siskakis demonstrated that $\left(C_{t}\right)_{t \geq 0}$ is strongly continuous on the Bergman space $A_{\alpha}^{p} \quad(1 \leq p<\infty$; $-1<\alpha<\infty)$ and the Dirichlet space $\mathscr{D}$ in $[7,8]$. Moreover, he showed that the infinitesimal generator $\Gamma$ of a semigroup of composition operators on these spaces is of the form $\Gamma f=G f^{\prime}$ with a certain domain. Refer [9-16] for more results of composition semigroups on other various spaces.

In this paper, we consider composition semigroups on weighted Bergman spaces $A_{\omega}^{p}$ with $\omega \in \widehat{\mathscr{D}}$. We will show that every $\left(C_{t}\right)_{t \geq 0}$ is strongly continuous on $A_{\omega}^{p}$ if $1 \leq p<\infty$ and $\omega \in \widehat{\mathscr{D}}$. The corresponding infinitesimal generator $\Gamma$ of $\left(C_{t}\right)_{t \geq 0}$ and its point spectrum can also be identified. In addition, if the Denjoy-Wolff point of $\left(\varphi_{t}\right)_{t \geq 0}$ belongs to $\mathbb{D}$, then we can also characterize the compactness of resolvent operator $R(\lambda, \Gamma)$ of $\Gamma$, provided $\lambda$ belongs to the resolvent set of $\Gamma$.

Throughout the paper, the symbol $A \approx B$ means that $A \lesssim B \lesssim A$. We say that $A \lesssim B$ if there exists a constant $C$ such that $A \leq C B$.

\section{Strongly Continuous Composition Semigroup}

We need more information about $\left(\varphi_{t}\right)_{t \geq 0}$ before presenting our results.

Assume a semigroup $\left(\varphi_{t}\right)_{t \geq 0}$ consisting of analytic selfmaps of $\mathbb{D}$ with infinitesimal generator $G$ and Denjoy-Wolff point $b$. In general, $\left(\varphi_{t}\right)_{t \geq 0}$ can be classified into two classes: $b \in \mathbb{D}$ and $b \in \partial \mathbb{D}$, the boundary of $\mathbb{D}$. In particular, there exists a unique univalent function $h: \mathbb{D} \longrightarrow \mathbb{C}$, called Koenigs function, such that

(1) If $b \in \mathbb{D}$, then $h(b)=0, h^{\prime}(b)=1$ and

$$
\left(h^{\circ} \gamma_{b}\right)\left(\varphi_{t}(z)\right)=e^{G^{\prime}(b) t}\left(h^{\circ} \gamma_{b}\right)(z) \quad \text { for } z \in \mathbb{D} \text { and } \geq 0 \text {, }
$$

where $\gamma_{b}(z)=z-b / 1-\bar{b} z$. Moreover,

$$
G(z)\left(h^{\circ} \gamma_{b}\right)(z)=G^{\prime}(b)\left(h^{\circ} \gamma_{b}\right)(z), \quad z \in \mathbb{D} \text {. }
$$

(2) If $b \in \partial \mathbb{D}$, then $h(0)=0$ and

$$
h\left(\varphi_{t}(z)\right)=h(z)+G(0) t \quad \text { for } z \in \mathbb{D} \text { and } t \geq 0 .
$$

Moreover,

$$
h^{\prime}(z) G(z)=G(0), \quad z \in \mathbb{D} .
$$

Lemma 1 shows that every composition operator is bounded on $A_{\omega}^{p}$ if $0<p<\infty$ and $\omega \in \widehat{\mathscr{D}}$. The proof can be easily obtained by a simple combination of Theorem 15 and (4.7) in [17].

Lemma 1 (see [17]). Let $0<p<\infty$ and $\omega \in \widehat{\mathscr{D}}$, and let $\varphi$ be an analytic self-map of $\mathbb{D}$. Then, the composition $C_{\varphi}$ is bounded on $A_{\omega}^{p}$. Moreover, there exist constants $\eta=\eta(\omega)>1$ and $C=C(\eta, \omega, p)$ such that

$$
\left\|C_{\varphi}\right\| \leq C\left(\frac{1+|\varphi(0)|}{1-|\varphi(0)|}\right)^{\eta} .
$$

Now, we are ready to show our results. For $f \in \mathscr{H}(\mathbb{D})$ and $0<r<1$, set

$$
\begin{aligned}
M_{p}(r, f) & =\left(\frac{1}{2 \pi} \int_{0}^{2 \pi}\left|f\left(r e^{i \theta}\right)\right|^{p}\right)^{1 / p}, \quad 0<p<\infty, \\
M_{\infty}(r, f) & =\sup _{|z=r|}|f(z)| .
\end{aligned}
$$

Theorem 1. Let $1 \leq p<\infty$ and $\omega \in \widehat{\mathscr{D}}$. Suppose $\left(\varphi_{t}\right)_{t \geq 0}$ is a semigroup of analytic self-maps of $\mathbb{D}$ with infinitesimal generator $G$. Then, the induced composition semigroup $\left(C_{t}\right)_{t \geq 0}$ defined in (4) is strongly continuous on $A_{\omega}^{p}$ with infinitesimal generator $\Gamma$ :

$$
\Gamma f=G f^{\prime}
$$

on its domain

$$
D(\Gamma)=\left\{f \in A_{\omega}^{p}: G f^{\prime} \in A_{\omega}^{p}\right\} .
$$

Moreover, $\left(C_{t}\right)_{t \geq 0}$ is uniformly continuous on $A_{\omega}^{p}$ if and only if $\left(\varphi_{t}\right)_{t \geq 0}$ is trivial.

Proof. Since $\omega \in \widehat{\mathscr{D}}$, the polynomials are dense in $A_{\omega}^{p}$. Therefore, for any $f \in A_{\omega}^{P}$, there exists a sequence of polynomials $\left\{P_{n}\right\}$ such that $\lim _{n \longrightarrow \infty}\left\|f-P_{n}\right\|_{A_{\omega}^{p}}=0$. It follows triangle inequality that

$$
\begin{aligned}
\left\|C_{t} f-f\right\|_{A_{\omega}^{p}} & \leq\left\|C_{t} f-C_{t} P_{n}\right\|_{A_{\omega}^{p}}+\left\|C_{t} P_{n}-P_{n}\right\|_{A_{\omega}^{p}}+\left\|f-P_{n}\right\|_{A_{\omega}^{p}} \\
& \leq\left(\left\|C_{t}\right\|+1\right)\left\|f-P_{n}\right\|_{A_{\omega}^{p}}+\left\|C_{t} P_{n}-P_{n}\right\|_{A_{\omega}^{p} .}
\end{aligned}
$$

According to Lemma 1, we know that $\sup _{t \in[0,1]}\left\|C_{t}\right\|<\infty$. Therefore, to prove $\lim _{t \rightarrow 0^{+}}\left\|C_{t} f-f\right\|_{A_{\omega}^{p}}=0$, it suffices to prove $\lim _{t \longrightarrow 0^{+}}\left\|C_{t} P-P\right\|_{A_{\omega}^{p}}=0$ for each polynomial $P$. Equivalently, we only need to show that for each $n \geq 0, \quad \lim _{t \longrightarrow 0^{+}}\left\|\left(\varphi_{t}\right)^{n}-e_{n}\right\|_{A_{\omega}^{p}}=0$, where $e_{n}(z)=z^{n}$, while it can be easily obtained by Lebesgue-dominated convergence theorem. Thus, $\left(C_{t}\right)_{t \geq 0}$ is strongly continuous on $A_{\omega}^{p}$.

By definition, the domain of $\Gamma$ is 


$$
D(\Gamma)=\left\{f \in A_{\omega}^{p}: \lim _{t \longrightarrow 0^{+}} \frac{C_{t}(f)-f}{t} \text { exists in } A_{\omega}^{p}\right\} .
$$

Let, now, $D=\left\{f \in A_{\omega}^{p}: G f^{\prime} \in A_{\omega}^{p}\right\}$. We are going to show that if $f \in D(\Gamma)$, then $G f^{\prime} \in A_{\omega}^{p}$. Indeed, if $f \in D(\Gamma)$, then $\Gamma(f) \in A_{\omega}^{p}$ and

$$
\lim _{t \longrightarrow 0^{+}}\left\|\frac{C_{t} f-f}{t}-\Gamma(f)\right\|_{A_{\omega}^{p}}=0 .
$$

Since for a fixed $0<r<1$, the well-known inequality $M_{\infty}(r, f) \leqslant M_{p}(1+r / 2, f)(1-r)^{-1 / p}$ yields

$$
M_{\infty}^{p}(r, f) \leqslant \frac{\|f\|_{A_{\omega}^{p}}^{p}}{(1-r) \widehat{\omega}(r)} .
$$

Convergence in the norm of $A_{\omega}^{p}$ implies the pointwise convergence. Therefore, for every $z \in \mathbb{D}$,

$$
\begin{aligned}
\Gamma f(z) & =\lim _{t \longrightarrow 0^{+}} \frac{f\left(\varphi_{t}(z)\right)-f(z)}{t} \\
& =\left.\lim _{t \longrightarrow 0^{+}} \frac{f\left(\varphi_{t}(z)\right)-f\left(\varphi_{0}(z)\right)}{t} \frac{\partial\left(f\left(\varphi_{t}(z)\right)\right)}{\partial t}\right|_{t=0} \\
& =G(z) f^{\prime}(z) .
\end{aligned}
$$

That is, $G(z) f^{\prime}(z)=\Gamma f(z) \in A_{\omega}^{p}$ and $D(\Gamma) \subseteq D$. On the other hand, for $\lambda \in \rho(\Gamma)$, the resolvent set of $\Gamma$, we have

$$
\begin{aligned}
D & =\left\{f \in A_{\omega}^{p}: G f^{\prime} \in A_{\omega}^{p}\right\}=\left\{f \in A_{\omega}^{p}: G f^{\prime}-\lambda f \in A_{\omega}^{p}\right\} \\
& =R(\lambda, \Gamma),
\end{aligned}
$$

where $R(\lambda, \Gamma)=(\lambda I-\Gamma)^{-1}$ is the resolvent operator of $\Gamma$. Since $R(\lambda, \Gamma)\left(A_{\omega}^{p}\right) \subseteq D(\Gamma), D \subseteq D(\Gamma)$. Hence, $D=D(\Gamma)$.

If $\left(C_{t}\right)_{t \geq 0}$ is uniformly continuous on $A_{\omega}^{p}$, then the infinitesimal generator $\Gamma$ is bounded on $A_{\omega}^{p}$. To show $\left(\varphi_{t}\right)_{t \geq 0}$ is trivial, it is equivalent to show $G \equiv 0$. To the end, we may consider polynomials $e_{n}(z)=z^{n}$. Then, $\Gamma\left(e_{n}\right)=n G e_{n-1}$, and taking $n=1$, we see that $G \in A_{\omega}^{p}$. Since $\Gamma$ is bounded on $A_{\omega}^{p}$, for $n \geq 1$, we have $\left\|\Gamma e_{n}\right\| \leq\left\|e_{n}\right\|$, that is,

$$
n^{p} \int_{0}^{1} M_{p}^{p}(r, G) r^{(n-1) p+1} \omega(r) \mathrm{d} r \leqslant \int_{0}^{1} r^{n p+1} \omega(r) \mathrm{d} r .
$$

It follows that $n^{p} M_{p}^{p}(1 / 2, G) \leq 1$. Since $G \in A_{\omega}^{p}$, we have $M_{p}(1 / 2, G)=0$. Thus, $G \equiv 0$. The proof is complete.

Theorem 2. Let $1 \leq p<\infty$ and $\omega \in \widehat{\mathscr{D}}$. Suppose $\left(\varphi_{t}\right)_{t \geq 0}$ is a semigroup of analytic self-maps of $\mathbb{D}$ with Denjoy-Wolff point $b$, infinitesimal generator $G$, and associated Koenigs function $h$.

(i) If $b \in \mathbb{D}$, then $P \sigma(\Gamma) \subseteq\left\{k G^{\prime}((b): k=0,1,2, \ldots\}\right.$. And, $k G^{\prime}(b) \in P \sigma(\Gamma)$ if and only if $\left(h^{\circ} \gamma_{b}\right)^{k} \in A_{\omega}^{p}$.

(ii) If $b \in \partial \mathbb{D}$, then $P \sigma(\Gamma)=\left\{\lambda G(0): e^{\lambda h(z)} \in A_{\omega}^{p}\right\}$.

Proof. (i) By (7),

$$
G(z)=G^{\prime}(b) \frac{\left(h^{\circ} \gamma_{b}\right)}{\left(h^{\circ} \gamma_{b}\right)}
$$

Now, suppose $f \in \mathscr{H}(\mathbb{D})$ and $\lambda \neq 0$ such that $\Gamma f=G f^{\prime}=\lambda f$. Then,

$$
\frac{f^{\prime}}{f}=\lambda \frac{\left(h^{\circ} \gamma_{b}\right)^{\prime}}{G^{\prime}(b)\left(h^{\circ} \gamma_{b}\right)} \text {. }
$$

Pick $r$ such that $|b|<r<1$ and $f$ has no zeros on $|z|=r$. We have

$\frac{1}{2 \pi i} \int_{|z|=r} \frac{f^{\prime}(z)}{f(z)} \mathrm{d} z=\frac{\lambda}{G^{\prime}(b)} \frac{1}{2 \pi i} \int_{|z|=r} \frac{\left(h^{\circ} \gamma_{b}\right)^{\prime}(z)}{\left(h^{\circ} \gamma_{b}\right)(z)} \mathrm{d} z$.

From this and the argument principle, it follows that $\lambda / G^{\prime}(b)=k$, a nonnegative integer, which shows the first part of (i). Also, notice that the differential equation

$$
f^{\prime}(z)=k \frac{\left(h^{\circ} \gamma_{b}\right)^{\prime}(z)}{\left(h^{\circ} \gamma_{b}\right)(z)} f(z)
$$

has solution $f(z)=c\left(h^{\circ} \gamma_{b}\right)^{k}$, where $c \neq 0$. This shows the second part of (i).

(ii) By (10), we have $G(z)=G(0) / h^{\prime}(z)$. If $f(z)=e^{\lambda h(z)} \in A_{\omega}^{p}$, then $\Gamma(f)=\lambda G(0) f$, so $\lambda G(0) \in P \sigma(\Gamma)$. Conversely, if $\Gamma f=G^{\prime} f=\lambda G(0) f$, then $f^{\prime}(z)=\lambda h^{\prime}(z) f(z)$, which follows that $f(z)=c e^{\lambda h(z)}$, where $c \neq 0$. The proof is complete.

\section{Resolvent Operator}

In [18], Siskakis characterized the compactness of $R(\lambda, \Gamma)$ on the Hardy space $H^{p}$ and the weighted Bergman space $A_{\alpha}^{p}$ if the Denjoy-Wolff point of $\left(\varphi_{t}\right)_{t>0}$ is in the interior of $\mathbb{D}$. In this section, we will consider the compactness of $R(\lambda, \Gamma)$ on $A_{\omega}^{p}$ with $\omega \in \widehat{\mathscr{D}}$.

For $\alpha \geq 1$ and $\omega \in \widehat{\mathscr{D}}, \mathscr{C}^{\alpha}\left(\omega^{*}\right)$ consists of all $f \in \mathscr{H}(\mathbb{D})$ such that

$$
\|f\|_{\mathscr{C}^{\alpha}\left(\omega^{*}\right)}^{2}=|g(0)|^{2}+\sup _{I \epsilon \partial \mathbb{D}} \frac{\int_{S(I)}|f(z)|^{2} \omega^{*}(z) d A(z)}{(\omega(S(I)))^{\alpha}}<\infty .
$$

$\mathscr{C}_{0}^{\alpha}\left(\omega^{*}\right)$ consists of all $f \in \mathscr{H}(\mathbb{D})$ such that

$$
\lim _{|I| \longrightarrow 0} \frac{\int_{S(I)}\left|f^{\prime}(z)\right|^{2} \omega^{*}(z) d A(z)}{(\omega(S(I)))^{\alpha}}=0,
$$

where $S(I)=\left\{r e^{i t} \in \mathbb{D}: e^{i t} \in I, 1-|I| \leq r<1\right\}$ is Carleson square associated with $I$ and $\omega^{*}(z)=\int_{|z|}^{1} \log s /|z| s d \omega(s)$ for all $z \in \mathbb{D} /\{0\}$. Refer $[1,2]$ for more information about these spaces.

To prove the main result in this section, we need the following lemma, which characterizes the boundedness and 
compactness of integral operator $V_{g}$ on $A_{\omega}^{p}$. Here, for a $g \in \mathscr{H}(\mathbb{D}), V_{g}$ is defined as

$$
V_{g}(f)(z)=\frac{1}{z} \int_{0}^{z} f(\zeta) g^{\prime}(\zeta) f \zeta, \quad z \in \mathbb{D}, f \in \mathscr{H}(\mathbb{D}) .
$$

Lemma 2 (see $[1,2])$. Let $0<p<\infty, \omega \in \widehat{\mathscr{D}}$, and $g \in \mathscr{H}(\mathbb{D})$. Then,

(i) $V_{g}$ is bounded on $A_{\omega}^{p}$ if and only if $g \in \mathscr{C}^{1}\left(\omega^{*}\right)$

(ii) $V_{g}$ is compact on $A_{\omega}^{p}$ if and only if $g \in \mathscr{C}_{0}^{1}\left(\omega^{*}\right)$

Lemma 3 is critical to our result.

Lemma 3. Let $1 \leq p<\infty$ and $\omega \in \widehat{\mathscr{D}}$, and let $\left(\varphi_{t}\right)_{t \geq 0}$ be $a$ nontrivial semigroup of self-maps on $\mathbb{D}$ with Denjoy-Wolff point 0 , infinitesimal generator $G$, and Koenigs function $h$. Suppose $\left(C_{t}\right)_{t \geq 0}$ is the corresponding composition semigroup on $A_{\omega}^{p}$, with the generator $\Gamma$. Then, for all $\lambda \in \rho(\Gamma)$, the resolvent operator of $\Gamma$ has the following representation:

$$
\begin{aligned}
R(\lambda, \Gamma) f(z)= & -\frac{1}{G^{\prime}(0)} \frac{1}{(h(z))^{\lambda /-G^{\prime}(0)}} \\
& \cdot \int_{0}^{z} f(\zeta)(h(\zeta))^{\lambda /-G^{\prime}(0)-1} h^{\prime}(\zeta) \mathrm{d} \zeta .
\end{aligned}
$$

In particular, $-G^{\prime}(0)$ belongs to $\rho(\Gamma)$, and hence,

$$
R(-G(0), \Gamma) f(z)=-\frac{1}{G^{\prime}(0) h(z)} \int_{0}^{z} f(\zeta) h^{\prime}(\zeta) \mathrm{d} \zeta
$$

Proof. Let

$$
R:=-\frac{1}{G^{\prime}(0)} \frac{1}{(h(z))^{\lambda /-G^{\prime}(0)}} \int_{0}^{z} f(\zeta)(h(\zeta))^{\lambda /-G^{\prime}(0)-1} h^{\prime}(\zeta) \mathrm{d} \zeta .
$$

It is elementary to compute that $(\lambda I-\Gamma) R=R(\lambda I-\Gamma)=I$, which shows that $R$ is the resolvent operator of $\Gamma$. Since the Denjoy-Wolff point of $\left(\varphi_{t}\right)_{t \geq 0}$ is 0 , it is easy to see that $\operatorname{Re}\left(-G^{\prime}(0)\right) \geq 0$. If $\operatorname{Re}\left(-G^{\prime}(0)\right)>0$, by Lemma 1 , we have

$$
\omega_{0}:=\lim _{t \longrightarrow \infty} \frac{\log \left\|C_{t}\right\|}{t}=0 .
$$

So, $-G^{\prime}(0) \in \rho(\Gamma)$ by Theorem 1.10 in ([6], p42). If $-G^{\prime}(0)$ is a pure imaginary number, write $G(z)=-i \alpha z$,

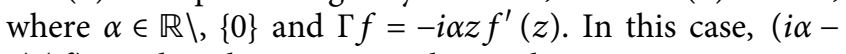
$\Gamma)(f)=g$ has the unique analytic solution

$$
f(z)=\frac{1}{i \alpha z} \int_{0}^{z} g(\zeta) \mathrm{d} \zeta .
$$

It is not difficult to see that the operator

$$
g \longrightarrow \frac{1}{i \alpha z} \int_{0}^{z} g(\zeta) \mathrm{d} \zeta
$$

is bounded on $A_{\omega}^{p}$. Hence, $-G^{\prime}(0)$ belongs to $\rho(\Gamma)$. The proof is complete.

Now, we are ready to prove the main result in this section.

Theorem 3. Let $1 \leq p<\infty$ and $\omega \in \widehat{\mathscr{D}}$. Suppose $\left(\varphi_{t}\right)_{t \geq 0}$ is a semigroup of analytic self-maps of $\mathbb{D}$ with Denjoy-Wolff point 0 , infinitesimal generator $G$, and associated Koenigs function $h$. Denote by $\Gamma$ the infinitesimal generator if the corresponding composition semigroup $\left(C_{t}\right)_{t \geq 0}$ on $A_{\omega}^{p}$ and denote by $R(\lambda, \Gamma)$ the resolvent operator for $\lambda \in \rho(\Gamma)$. Then, the following statements are equivalent:

(i) $R(\lambda, \Gamma)$ is compact on $A_{\omega}^{p}$

(ii) $\operatorname{logh}(z) / z \in \mathscr{C}_{0}^{1}\left(\omega^{*}\right)$

Proof. The well-known resolvent equation

$$
R(\lambda, \Gamma)-R(\mu, \Gamma)=(\mu-\lambda) R(\lambda, \Gamma) R(\mu, \Gamma) \quad \lambda, \mu \in \rho(\Gamma),
$$

shows that $R(\lambda, \Gamma)$ is compact on $A_{\omega}^{p}$ for all $\lambda \in \rho(\Gamma)$ if and only if it is compact for a certain $\lambda_{0} \in \rho(\Gamma)$. So, by Lemma 3, to prove the compactness of $R(\lambda, \Gamma)$, it is suffices to identify the compactness of $R\left(-G^{\prime}(0), \Gamma\right)$, or equivalently, the compactness of $R_{h}$ :

$$
R_{h}(f)(z)=\frac{1}{h(z)} \int_{0}^{z} f(\zeta) h^{\prime}(\zeta) \mathrm{d} \zeta
$$

To this end, we will use the technology mentioned in [18], which points out that $R_{h}$ can be decomposed as follows:

$$
M_{z} P_{h}=R_{h} M_{z}, Q_{h}=P_{h}+Q_{h} P_{h},
$$

where

$$
\begin{aligned}
M_{z} f(z) & =z f(z) \\
P_{h} f(z) & =\frac{1}{z h(z)} \int_{0}^{z} f(\zeta) \zeta h^{\prime}(\zeta) \mathrm{d} \zeta \\
Q_{h} f(z) & =\frac{1}{z} \int_{0}^{z} f(\zeta) \frac{\zeta h^{\prime}(\zeta)}{h(\zeta)} \mathrm{d} \zeta
\end{aligned}
$$

Moreover, it is elementary to see that

$$
Q_{h} f(z)=J(f)(z)+L_{h} M_{z}(f)(z),
$$

where $J(f)(z)=1 / z \int_{0}^{z} f(\zeta) \mathrm{d} \zeta$ and

$$
L_{h}(f)(z)=\frac{1}{z} \int_{0}^{z} f(\zeta)\left(\log \frac{h(\zeta)}{\zeta}\right)^{\prime} \mathrm{d} \zeta
$$

Since $R_{h}$ is bounded on $A_{\omega}^{p}, P_{h}$ is bounded on $A_{\omega}^{p}$ by (38). Furthermore, since $h$ is univalent and $h(0)=0$, it is well known that $\log (h(z) / z) \in \mathrm{BMOA}$. Hence, $\log (h(z) / z) \in \mathscr{C}^{1}\left(\omega^{*}\right)$. So by Lemma $2, L_{h}$ is bounded on $A_{\omega}^{p}$. Therefore, the compactness of $J$ and $L_{h}$ and (40) yield the boundedness of $Q_{h}$ on $A_{\omega}^{p}$. Consequently, (38) and (40) imply that $R_{h}$ is compact if and only if $L_{h}$ is compact if and 
only if $\log h(z) / z \in \mathscr{C}_{0}^{1}\left(\omega^{*}\right)$ by Lemma 2 . The proof is finished.

Remark 1. By [1], we immediately know that $\mathrm{BMOA} \subsetneq \mathscr{C}^{1}\left(\omega^{*}\right) \subsetneq \mathscr{B}$ and VMOA $\subsetneq \mathscr{C}_{0}^{1}\left(\omega^{*}\right) \subsetneq \mathscr{B}_{0}$. Therefore, in the above theorem, $\log h(z) / z \in \mathscr{C}_{0}^{1}\left(\omega^{*}\right)$ if and only if $\log h(z) / z \in \mathrm{VMOA}$ if and only if $\log h(z) / z \in \mathscr{B}_{0}$, which indicates that $R(\lambda, \Gamma)$ is compact on $A_{\omega}^{p}$ if and only if it is compact on the Hardy space $H^{p}$ if and only if it is compact on the classical Bergman space $A^{p}$ by Theorem 6.1 in [17]. This makes sense since $A_{\omega}^{p}$ induced by $\omega \in \widehat{\mathscr{D}}$ lies between $H^{p}$ and $A^{p}$.

It is well known that if $R(\lambda, \Gamma)$ is compact on $A_{\omega}^{p}$, then the spectrum $\sigma(\Gamma)$ of $\Gamma$ is only the point spectrum $P \sigma(\Gamma)$. By a simple combination of Theorems 2 and 3, we are in a position to depict the spectra of infinitesimal generators of some special composition semigroups. We end up the paper by showing some examples.

Example 1. For a $\operatorname{Rec} \geq 0$,consider the semigroup

$$
\varphi_{t}(z)=e^{-c t} z, \quad t \geq 0, z \in \mathbb{D},
$$

with infinitesimal generator $G(z)=-c z$ and Koenigs function $h(z)=z$. Apparently, its Denjoy-Wolff point is 0 and $h \in \mathscr{C}_{0}^{1}\left(\omega^{*}\right)$. Therefore, by Theorems 2 and 3 , the spectrum $\sigma(\Gamma)$ of infinitesimal generator $\Gamma$ of the corresponding composition semigroup $\left(C_{t}\right)_{t \geq 0}$ is

$$
\sigma(\Gamma)=\{-c k: k=k=0,1,2, \cdots\} .
$$

Example 2. Consider the semigroup

$$
\varphi_{t}(z)=1-(1-z)^{e^{-t}}, \quad t \geq 0, z \in \mathbb{D},
$$

with Denjoy-Wolff point 0 , infinitesimal generator $G(z)=-(1-z) \log 1 / 1-z$, and Koenigs function $h(z)=\log 1 / 1-z$. Similarly, the spectrum $\sigma(\Gamma)$ of infinitesimal generator $\Gamma$ of the corresponding composition semigroup $\left(C_{t}\right)_{t \geq 0}$ is

$$
\sigma(\Gamma)=\{-k: k=k=0,1,2, \cdots\} .
$$

\section{Data Availability}

No datasets were generated or analysed during the current study.

\section{Conflicts of Interest}

The author declares that there are no conflicts of interest.

\section{Acknowledgments}

This research was supported in part by the NNSF of China (Grant no. 11720101003).

\section{References}

[1] J. Á. Peláez, "Small weighted Bergman spaces," in Proceedings of the Summer School in Complex and Harmonic Analysis, and Related Topics, 2016.

[2] J. Á. Peláez and J. Rättyä, "Weighted Bergman spaces induced by rapidly increasing weights," Memoirs of the American Mathematical Society, vol. 227, p. 1066, 2014 vi+124pp.

[3] K. Zhu, Operator Theory in Function Spaces, American Mathematical Society, Providence, RI, USA, 2007.

[4] J. Á. Peláez and J. Rättyä, "Bergman projection induced by radial weight," https://arxiv.org/pdf/1902.09837.pdf.

[5] E. Berkson and H. Porta, "Semigroups of analytic functions and composition operators," The Michigan Mathematical Journal, vol. 25, no. 1, pp. 101-115, 1978.

[6] K. Engel and R. Nagel, A Short Course on Operator Semigroups, Springer, New York, NY, USA, 2006.

[7] A. G. Siskakis, "Semigroups of composition operators in Bergman spaces," Bulletin of the Australian Mathematical Society, vol. 35, no. 3, pp. 397-406, 1987.

[8] A. G. Siskakis, "Semigroups of composition operators on the Dirichlet space," Results in Mathematics, vol. 30, no. 1-2, pp. 165-173, 1996.

[9] O. Blasco, M. D. Contreras, S. Díaz-Madrigal, J. Martínez, and A. G. Siskakis, "Semigroups of composition operators in BMOA and the extension of a theorem of sarason," Integral Equations and Operator Theory, vol. 61, no. 1, pp. 45-62, 2008.

[10] O. Blasco, M. D. Contreras, S. Díaz-Madrigal, J. Martínez, M. Papadimitrakis, and A. G. Siskakis, "Semigroups of composition operators and integral operators in spaces of analytic functions," Annales Academiae Scientiarum Fennicae Mathematica, vol. 38, no. 1, pp. 67-89, 2013.

[11] M. Contreras and S. Díaz-Madrigal, "Fractional iteration in the disk algebra: prime ends and composition operators," Revista Matemática Iberoamericana, vol. 21, pp. 911-928, 2005.

[12] F. Gürbüz, "Some estimates for generalized commutators of rough fractional maximal and integral operators on generalized weighted morrey spaces," Canadian Mathematical Bulletin, vol. 60, no. 1, pp. 131-145, 2017.

[13] F. Gürbüz, "Generalized weighted morrey estimates for marcinkiewicz integrals with rough kernel associated with schrödinger operator and their commutators," Chinese Annals of Mathematics, Series B, vol. 41, no. 1, pp. 77-98, 2020.

[14] F. Gürbüz, "On the behaviors of rough multilinear fractional integral and multi-sublinear fractional maximal operators both on product Lp and weighted Lp spaces," International Journal of Nonlinear Sciences and Numerical Simulation, vol. 21, no. 7-8, pp. 715-726, 2020.

[15] F. Gurbuz, "Some inequalities for the multilinear singular integrals with Lipschitz functions on weighted Morrey spaces," Journal of Inequalities and Applications, pp. 1-10, 2020.

[16] K. J. Wirths and J. Xiao, "Recognizing $\{\$ \backslash$ scr Q $\backslash$ sb $\{p, 0\} \$\}$ functions per Dirichlet space structure," Bulletin of the Belgian Mathematical Society - Simon Stevin, vol. 8, no. 1, pp. 47-59, 2001.

[17] J. Á. Peláez and J. Rättyä, "Trace class criteria for Toeplitz and composition operators on small Bergman spaces," Advances in Mathematics, vol. 293, pp. 606-643, 2016.

[18] A. G. Siskakis, W. Y. Laramie, Semigroups of composition operators on spaces of analytic functions, a review," Studies on Composition Operators, vol. 213, pp. 229-252, Contemperary Mathematics, American Mathematical Society, Providence, RI, USA, 1998. 